doi:10.1016/S1473-3099(06)70603-X

Copyright (c) 2006 Elsevier Ltd All rights reserved.

\title{
The polio eradication effort has been a great success-let's finish it and replace it with something even better
}

\section{Dr Tjeerd G Kimman PhD ${ }^{a, *}$ and Hein Boot PhD}

a Laboratory for Vaccine-Preventable Diseases, National Institute of Public Health and the Environment, Bilthoven, Netherlands

* Correspondence to: Dr Tjeerd G Kimman, Laboratory for Vaccine-Preventable Diseases, National Institute of Public Health and the Environment, PO Box 1, 3720 BA Bilthoven, Netherlands. tel +313027423 30; fax +3130274 4449

\section{Summary}

The polio eradication campaign has greatly reduced the effects of this disease, but many new challenges have emerged. These challenges include the occurrence of polio outbreaks caused by wild-type polioviruses or circulating vaccine-derived polioviruses (cVDPVs) in areas where vaccination coverage is low, the existence of people who excrete poliovirus persistently, and the inability to know definitely that poliovirus has gone. As a result, there is uncertainty about if, when, and how we can end polio immunisation. In this article, we discuss several scenarios for the future of polio control. Because the emergence of cVDPVs necessitates discontinuing the use of live oral polio vaccine, we propose to strive towards a global coverage of near $100 \%$ vaccination against all major childhood infections using combination vaccines that contain inactivated poliovirus vaccine. Such a policy will present multiple challenges.

\section{Introduction}

In 1988 the World Health Assembly (WHA) launched the polio eradication effort, with the target of a polio-free world by the year 2000, a goal that was later postponed until 2005. The aim was to stop the transmission of poliovirus through vaccination, after which vaccination itself could be stopped. The eradication campaign is zealously led by the WHO, but at the end of 2005 the goal of eradication had still not been reached. The benefits of the campaign are, nonetheless, huge and indisputable. First, since 1988 the effects of polio, a lethal or lifelong crippling disease, have been reduced by more than $99 \%$ to 1265 cases worldwide in 2004 . In $1988,10 \%$ of the world's children lived in polio-free countries. Now $70 \%$ of them do so. Second, it has become clear that polio eradication is a feasible target, provided that immunisation coverage is kept high in every corner of the world. Indeed, serotype 2 poliovirus has been eradicated since 1999. Finally, although not its primary aim, the eradication effort has brought with it huge improvements in public-health infrastructure in the developing world by providing means to deliver vaccines and to diagnose infections.

During the process of polio eradication, we have faced problems that are yet to be solved, and it would be naive to close our eyes to them. Perhaps the most troublesome problem is the switch from a vaccinating to a non-vaccinating world once the virus is thought to have disappeared. At present, we do not know how 
and when we can safely stop the use of the live oral polio vaccine (OPV), the vaccine developed by Sabin, which is used in most parts of the world to halt or prevent the transmission of the virus. ${ }^{1}$

What was once thought to be a theoretical and perhaps remote risk ${ }^{2}$ has become real: the emergence of circulating vaccine-derived poliovirus (cVDPV) strains. These strains of poliovirus evolved from Sabin OPV strains through the acquisition of point mutations, with or without an exchange of parts of the viral genome with related non-polio enteroviruses. Although these strains are derived from vaccine viruses, they are able to transmit from person-to-person and cause disease that is indistinguishable from wild-type poliovirus. ${ }^{3}$ and 4 cVDPV strains develop in parts of the world where live OPV is used and where immunisation coverage is low. A reduction in immunisation coverage could occur after local eradication of the disease in an attempt to save money and the work involved in maintaining coverage.

Since the launch of the WHA eradication effort, outbreaks caused by cVDPVs have occurred in Egypt (1988-1993), Hispaniola (2000), the Philippines (2001), Madagascar (2002), China (2004), Indonesia, and in the Amish population in the USA (2005). The potential risk of cVDPV emergence has increased profoundly in recent years as wild poliovirus circulation has ceased in many parts of the world. The risk appears highest for type 2 OPV because of its apparent greater capacity than types 1 and 3 to spread to contacts. ${ }^{4}$ Experience shows that using OPV while immunisation coverage is not optimal in every part of the world is like playing with fire. The emergence of cVDPVs, therefore, necessitates stopping the use of OPV.

Other issues related to halting immunisation are the inability to know for sure that poliovirus has been eradicated, the existence of persistent shedders (immune compromised people who excrete the virus in their faeces for many years), and the safeguarding of remaining stocks of polioviruses in laboratories and vaccine factories worldwide.

\section{Oral polio vaccine discontinuation}

Although several scenarios have been discussed with regard to stopping OPV vaccination-ranging from global synchronised cessation ${ }^{5}$ to temporal replacement of OPV with the safe but more expensive inactivated polio vaccine (IPV)-the success of none of these approaches is guaranteed. Many rich countries have replaced OPV by IPV already, largely because OPV could cause vaccine-associated paralysis. Furthermore, it would be very desirable to stop the use of OPV, because of the risks associated with reversion to virulence. However, stopping the provision of OPV without keeping population immunity at a high level is not an option, because such a situation will pose a risk for the youngest individuals, who could develop paralysis as a result of cVDPVs, and could, ironically, necessitate the deliberate reintroduction of OPV itself to combat such epidemics.

Unfortunately, the discussion on how to stop OPV vaccination coincides with many setbacks in the eradication itself. Poliovirus serotype 1 caused a very serious and large outbreak during 2004 in western and central Africa (spreading from Nigeria where vaccination was refused for political or theological reasons, or fear of deliberate contamination of the vaccine with HIV or infertility agents). The same virus travelled in 2005 to Yemen, Saudi Arabia, and Indonesia (probably aided by Muslim pilgrims returning from the Hajj or migrant workers). More than 1500 children have become paralysed. The spread of the virus is even worse than 
observed clinically, because only a few infected children become clinically ill, and not all cases are notified. Indeed, poliovirus can spread unnoticed within a population, since only one in several hundred infections results in paralysis. This ratio varies according to serotype, and is highest for serotype 3 (estimated at between 4000/1 and 500/1). The lowest ratio is detected for serotype 1 (between $60 / 1$ to $175 / 1$ ); intermediate values have been found for serotype 2 (1000/1 or higher). ${ }^{6}$ and 7

\section{Vaccination campaign scenarios}

In our opinion, experience shows that poliovirus will not be eradicated unless vaccination coverage is close to $100 \%$ (ie, $>95 \%$ ) in every corner of the world. Most recent polio outbreaks, either caused by wild-type poliovirus strains or cVDVPs, occurred in regions with insufficient routine immunisation levels, which gave the virus good opportunities to spread upon its (re)introduction. This was the case in Hispaniola, Niger, Nigeria, Burkina Faso, Central African Republic, Chad, Côte d'Ivoire, Mali, Sudan, Yemen, Angola, and Indonesia (table).

Unfortunately, national immunisation days were stopped recently in many poliofree countries to focus vaccination efforts towards endemic areas, leaving many children unprotected. ${ }^{11}$ The recent outbreaks show that vaccinating against the virus away from endemic areas while enhancing the number of susceptible individuals elsewhere is too dangerous. Although polio eradication seems to be nearly reached, there is serious doubt as to whether this will ever happen.

\begin{tabular}{|c|c|}
\hline & $\begin{array}{l}\text { Proportion of unimmunised or } \\
\text { underimmunised people (\%) }\end{array}$ \\
\hline \multicolumn{2}{|c|}{ Coverage in countries before recent polio transmission } \\
\hline Angplo & $52 \%$ \\
\hline Eukina faso & $177 \%$ \\
\hline CentralAfical Republic & $6 \mathrm{cs}$ \\
\hline Chad & 5220 \\
\hline Chira: & $9 \mathrm{~s}$ \\
\hline Côle d'Wulte & $\triangle 6 \%$ \\
\hline Hispaniola (affected arcas) & $6093 \%$ \\
\hline Indrinerla & 30 \% \\
\hline Madajascar & $42 \%$ \\
\hline Mall & 396 \\
\hline Higer & 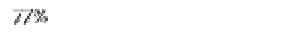 \\
\hline Hiugris & $68 \%$ \\
\hline Milippines" & $23 \%$ \\
\hline sudan & $50 \%$ \\
\hline yenen & $34 \times$ \\
\hline \multicolumn{2}{|c|}{ Coverage in 2004 in some countries at risk } \\
\hline Afghtanistan & $34 \%$ \\
\hline Anlupla & 43 \\
\hline Bolvis & $16 \mathrm{~m}$ \\
\hline Camernos & $2 A m$ \\
\hline Gongu Repubic & 33\% \\
\hline Equatorial Gu nea & $54 \%$ \\
\hline Esthizpia & $35 \%$ \\
\hline Gabon & $84 \%$ \\
\hline Ceorgis & $34 \%$ \\
\hline Guinea & $32 \mathrm{~F}$ \\
\hline Likeria & $67 \%$ \\
\hline Mozanbique & 35\% \\
\hline $\begin{array}{l}\text { Werherhands } \\
\text { (local communities inthe viltsebelt? }\end{array}$ & $35 \%$ \\
\hline Fapue New Gunea & $6.4 \mathrm{~m}$ \\
\hline Simra Låne & $39 \%$ \\
\hline Somalia & $70 \mathrm{~s}$ \\
\hline Sudan & $21 \%$ \\
\hline
\end{tabular}

Table.

Polio vaccination coverage $\mathrm{e}^{8,9}$ and 10

* Outbreaks caused by circulating vaccine-derived polioviruses.

WHO organises emergency catch-up vaccination campaigns in response to every new outbreak. The emergency campaigns, therefore, travel slowly behind the virus, rather than preventing outbreaks occurring in the first place. In some cases, implementation of an emergency vaccination campaign after the detection of an outbreak can take months (eg, Indonesia, 2005), during which time the virus can continue to spread. New polio cases even occurred in Indonesia after the second vaccination round. Catch-up vaccination campaigns can prevent polio in some children and help stop population spread, but they are not very economical because all children in a targeted area are vaccinated, irrespective of whether they need it or not. Thus, catch-up vaccination campaigns come too late and at too much cost.

This situation has serious consequences. First, the eradication of polio is at stake. 
Second, the emergency vaccination campaigns in response to outbreaks require much additional money, which the funding partners (eg, WHO, EU, UNICEF, US Centers for Disease Control and Prevention, Rotary International) have to raise. We do not know how long they will be prepared to do so. Third, the efforts for the eradication campaign compete with other regular public-health provisions, including routine immunisations. Funding partners, field and laboratory workers, and "eradicators" must not get tired or demoralised. As mentioned, the polio eradication has brought many benefits, but now that huge amounts of money are being spent to eradicate poliovirus fully, the opposite might occur.

Is there a solution to these questions and dilemmas? We propose a drastic change in vaccination policy; to shift the emphasis of worldwide efforts to eradicate poliovirus fully towards reaching a global coverage of near $100 \%$ routine immunisation against the major childhood infections, including diphtheria, pertussis, tetanus, Haemophilus influenzae type b (Hib), measles, rubella, hepatitis B, and, of course, polio. For that purpose, children should be vaccinated with combination vaccines containing IPV instead of OPV. Clearly, this goal can only be reached in the long term (10-20 years or more), with great effort, and by gradually replacing OPV.

Is this a realistic goal; what will it bring, and what will it cost? Obviously, such a goal will help defeat more vaccine-preventable diseases. An estimated 30 million children each year still lack proper vaccination. ${ }^{12}$ Every year 2-3 million children die from vaccine-preventable diseases, including 600000 from measles, 200000 from tetanus, 300000 from pertussis, and 390000 from Hib; 600000 people die from the consequences of a hepatitis $B$ infection acquired during childhood every year.

A multifaceted approach is also required to further the control of poliovirus itself. We consider the current transmission of poliovirus as evidence that polio eradication without near $100 \%$ routine vaccination coverage will not succeed. In many parts of the developed world-eg, the Netherlands and Sweden-polio has been eradicated through the routine use of IPV without much additional effort. Although infection of IPV-vaccinated people has been documented, IPV induces sufficient herd immunity to interrupt and prevent transmission, as experienced in France, the USA, and the Netherlands. ${ }^{13}$ and 14 Thus, polio eradication could come at little or no additional cost above that of routine childhood vaccination with IPV. Additional research is needed to determine if this could also occur in developing countries using the presently available IPV, or whether the dose, composition, or vaccination scheme must be adjusted to reach that purpose. A promising sign of IPV's current effectiveness (albeit in developed parts of world) is the lack of polio in countries using IPV for their routine vaccination programmes, despite the wide spread of the virus in Africa and Asia in 2004 and 2005. Experimental data on IPV's effectiveness in reducing poliovirus transmission are encouraging, suggesting that IPV-vaccinated communities are at least partly protected from the spread of poliovirus. ${ }^{15}$ Furthermore, enhancement of IPV potency could lead to even higher levels of mucosal immunity and stop transmission of wild-type poliovirus. Research on optimal and safe polio vaccination should, therefore, continue until a safe and effective scheme is in place everywhere. Even if the current policy leads to eradication of polio, routine polio vaccination is still subsequently required to enhance and maintain high levels of immunity to stop the spread of OPV strains and the emergence of cVDPVs.

When we focus our attention on raising routine vaccination coverage, we must not worsen the polio situation. OPV is, therefore, still required to prevent polio outbreaks and raise immunity levels until it can be replaced by routinely used 
combination vaccines that contain IPV. This is the only safe route towards discontinuing OPV use.

\section{Potential challenges}

Raising the worldwide coverage of IPV-containing combination vaccines to near $100 \%$ will result in additional challenges. First, immunisation practices that are successful in some places, such as developed countries, may not be successful elsewhere. Research has to optimise local immunisation practices-eg, neonatal or maternal vaccination, and new adjuvants that allow fewer vaccinations to be given. Development of new application methods that simplify injections, or that circumvent the need to maintain a cold chain to deliver vaccines would also bring huge advances.

Second, much more money is needed to build or scale up the infrastructure for the routine administration of injectable vaccines, in particular maternal and child health clinics. Many health workers need additional education to deliver injectable vaccines. Structures need to be developed to safeguard vaccine supply. These investments should not be prohibitive, since the effort will be cost-effective or even cost-saving in the end, and will lead to a sustainable infrastructure to prevent other childhood diseases-eg, vitamin A deficiency. Such an effort is not trivial in the poorest, most densely populated, and unhygienic places of the world that could also undergo political problems and war.

Building infrastructures for the effective provision of vaccines is not the only monetary stumbling block: IPV-containing combination vaccines are more expensive than OPV. The price per dose of OPV is approximately US $\$ 0 \cdot 10$. The increase in price for including IPV in combination vaccines varies widely, estimations ranging from $\$ 2.00$ for low-income countries, to $\$ 5.00$ for middleincome countries, and $\$ 10.00$ for high-income countries. ${ }^{16}$ The total expenditure for OPV cessation followed by IPV introduction is thus very sensitive to the cost of the vaccine, but would at least be $\$ 500$ million per year for vaccine costs only. In addition to the humanitarian benefits, there is also something to gain financially. Stopping OPV would save the costs associated with vaccine-derived poliovirus cases and cVDPV-associated outbreaks. Unfortunately, money spent on vaccination programmes could compete with money spent on, for example, AIDS or hunger programmes. Presumably the end goal (100\% coverage against the major vaccine-preventable diseases) might not be reached without intermediate stages-eg, raising immunity levels to polio and measles first.

Third, the production capacity of childhood vaccines, especially IPV, has to be raised considerably, preferably with more efficient and cheaper production methods. This costs money, but an increase in the number of vaccine doses sold might help to lower vaccine prices. Currently, the maximum IPV production capacity is estimated to be 240 million doses annually, which should be raised to more than 540 million doses for routine coverage in developed and developing countries. ${ }^{17}$

Raising coverage of routine immunisation programmes that include IPV requires more endurance than needed for achieving a quick and visible health gain. Many scientific, financial, infrastructural, logistical, and political issues must, therefore, be solved. Routine vaccination must not stop during the civil wars that have dogged the OPV vaccination campaigns. One might argue that implementing routine IPV vaccination to all children in the world is too expensive and not a realistic goal. If so, this would create dilemmas that are difficult to solve. Because insufficient population immunity could result in the spread of wild-type poliovirus 
and the emergence of cVDPVs, we do not consider ending the use of OPV to be an option, without first replacing it with IPV. If IPV vaccination is indeed too expensive, impractical, and beyond the reach of many developing countries in the coming years, then, as a second choice option only, it would be prudent to continue the use of OPV, raise its coverage to $100 \%$, and then, if circumstances allow, to slowly replace OPV with IPV. This option would mean that we do not get rid of OPV quickly, or even that we have to continue its use for ever. However, pursuit of this scenario might be realistic if it helps to improve routine vaccination against a wide range of childhood diseases.

The challenges we face are not scant, but creativity, wisdom, and ambition can achieve much in 10 years. The coming years could be a transition period from polio eradication to improved routine prevention of childhood diseases. If we succeed, we will probably have eradicated all or almost all polioviruses (even to the point that there is no doubt that IPV vaccination can be stopped) and associated paralysis, we will have prevented many more diseases than polio alone, and we will have an infrastructure that can be used to prevent other diseases. 
The Lancet Infectious Diseases Volume 6, Issue 10 , October 2006, Pages 675-678

\section{References}

1 L Roberts, The exit strategy, Science 303 (2004), pp. 1969-1971.

2 TG Kimman, MP Koopmans and HG van der Avoort, Ending polio immunization, Science 279 (1998), pp. 788-789.

$3 \mathrm{HJ}$ Boot, DTJ Kasteel, A-M Buisman and TG Kimman, Excretion of wild-type and vaccine-derived poliovirus in the feces of poliovirus receptor-transgenic mice, J Virol 77 (2003), pp. 6541-6545.

4 OM Kew, PF Wright and VI Agol et al., Circulating vaccine-derived polioviruses: current state of knowledge, Bull World Health Organ 82 (2004), pp. 16-23.

5 RB Aylward, RW Sutter and DL Heymann, Policy. OPV cessation-the final step to a "polio-free" world, Science 310 (2005), pp. 625-626.

$6 \mathrm{JL}$ Melnick and N Ledinko, Development of neutralizing antibodies against the three types of poliomyelitis during an epidemic period, Am J Hyg 58 (1953), pp. 207-222.

7 TB Turner, DH Hollander, S Buckley, UP Kokko and CP Winsor, Age, incidence and seasonal development of neutralizing antibodies to Lansing poliomyelitis virus, Am J Hyg 52 (1950), pp. 323347. Abstract-MEDLINE | Order Document

8 F Abbink, PJ Oomen, SLN Zwakhlas, HE de Melker and A Ambler-Huiskens, Immunization coverage in the Netherlands as at 1 January 2004, RIVM, Bilthoven, Netherlands (2005) 210021003/2005.

$90 \mathrm{Kew}, \mathrm{V}$ Morris-Glasgow and M Landaverde et al., Outbreak of poliomyelitis in Hispaniola associated with circulating type 1 vaccine-derived poliovirus, Science 296 (2002), pp. 356-359.

10 WHO, Immunization, surveillance, assessment, and monitoringhttp://www.who.int/immunization_monitoring/en/ (accessed December 1, 2005).

11 L Roberts, Polio: the final assault?, Science 303 (2004), pp. 1960-1968.

12 GAVI alliance, GAVI alliance general informationhttp://www.vaccinealliance.org (accessed Dec 1, 2005).

13 DJ Malvy and J Drucker, Elimination of poliomyelitis in France: epidemiology and vaccine status, Public Health Rev 21 (1997), pp. 41-49.

14 PM Oostvogel, JK van Wijngaarden and HG Van der Avoort et al., Poliomyelitis outbreak in an unvaccinated community in the Netherlands, 1992-93, Lancet 344 (1994), pp. 665-670.

15 M Laassri, K Lottenbach and R Bekshe et al., Effect of different vaccination schedules on excretion of oral poliovirus vaccine strains, J Infect Dis 192 (2005), pp. 2092-2098.

16 N Sangrujee, VM Caceres and SL Cochi, Cost analysis of post-polio certification immunization policies, Bull World Health Organ 82 (2004), pp. 9-15.

17 Milstein J. IPV production capacity, costs and containment (presentation). 6th meeting of the Technical Consultative Group on Polio Eradication; Geneva, Switzerland; May 7-10, 2001. 